\title{
An Fuzzy Petri Net Model of Accident Evolution Process of Coal and Gas Outburst
}

\author{
Li Ma \\ Communication and Information Engineering College \\ Xi'an University of Science and Technology \\ Xi'an, China \\ e-mail:maryraining@hotmail.com
}

\begin{abstract}
This article has proposed an accident evolution model of coal and gas outburst on the basis of fuzzy production rules, because the coal and gas outburst accident has the characteristic of fuzziness and uncertainty. Firstly, adverse searching method is used to reduce the initial model so as to decrease the size of the inference network and accelerate reasoning rate. And then fuzzy inference is carried out by use of parallel inference algorithms of great algebra in order to ensure more accurate infer conclusions. At the end of this article, the validity of the model is verified by an engineering example. From the conclusion, it can be found that this model can well simulate the status and role of a number of risk factors during the whole coal and gas outburst accident evolution process.
\end{abstract}

Keywords-coal and gas outburst; Fuzzy Petri Net (FPN); accident evolution process; fuzzy reasoning; adverse searching tactics

\section{INTRODUCTION}

Coal and gas outburst is a disaster occurred in the mining process of coal mine, which restricting the development of China coal industry and threatening to the safety of operating personnel ${ }^{[1]}$. There are many hazardous conditions hidden in the coal mine underground mining production process may lead to gas outburst accident ${ }^{[2]}$.

Therefore, in the premise of comprehensive consideration of coal and gas outburst risk evolution characteristics, this article has proposed an Accident Evolution Model of Coal and Gas Outburst on the Basis of Fuzzy Petri Net (AEMCGO-FPN). The model integration of knowledge representation and reasoning can give the whole process of the accident a structuring and visualizing description, which can help people recognize and understand the reason of the nature of accident and the occurrence process.

\section{THEORY OF FUZZY PETRI NET}

Fuzzy Petri Net (FPN) has two kinds of node: Place and Transition, and there are arcs connecting place and transition, where place denoted by a circle and transition denoted by the vertical line. When FPN is used in the fuzzy inference, an inference rule is denoted by a transition, and the evidence in inference rule is denoted by place. Each place has a Token value indicated the certainty factor of evidence, and each transition with corresponding Certainty Factor (CF)

National Natural Science Foundation of China (50874089

Xi'an University of Science and Technology Foundation (201162). value indicated the degree of confidence of inference rule. According to Reference ${ }^{[3]}$, in this article FPN can be defined as follows.

Fuzzy Petri Net includes four parts: Place, Transition, Certainty Factor、threshold values, which can be defined as a seven tuple.

Definition 1

$\mathrm{FPN}=(\mathrm{P}, \mathrm{T}, \mathrm{W}, \mathrm{CF}, \mathrm{I}, \mathrm{O}, \lambda)$

Where:

$\mathrm{P}=\left\{\mathrm{p}_{1}, \mathrm{p}_{2}, \ldots, \mathrm{p}_{\mathrm{n}}\right\}, \mathrm{P}$ is the finite set of the place;

$\mathrm{T}=\left\{\mathrm{t}_{1}, \mathrm{t}_{2}, \ldots, \mathrm{t}_{\mathrm{n}}\right\}, \mathrm{T}$ is the finite set of the transition;

$\mathrm{W}=\left(\mathrm{W}_{1}, \mathrm{~W}_{2}, \ldots, \mathrm{W}_{\mathrm{n}}\right)^{\mathrm{T}}, \mathrm{W}$ is the initial certainty factor of place $\mathrm{P}_{\mathrm{i}}, \mathrm{W} \in[0,1](\mathrm{i}=1,2, \ldots, \mathrm{n})$;

$\mathrm{CF}=\operatorname{diag}\left(\mathrm{CF}_{1}, \mathrm{CF}_{2}, \ldots, \mathrm{CF}_{\mathrm{m}}\right), \mathrm{CF}_{\mathrm{j}}$ is the certainty factor of transition $\mathrm{t}_{\mathrm{j}}, \mathrm{CF}_{\mathrm{j}} \in[0,1](\mathrm{j}=1,2, \ldots, \mathrm{m})$;

$\mathrm{I}=\left(\delta_{\mathrm{ij}}\right)$ is the rules input matrix from place to transition, $\delta_{\mathrm{ij}}$ $\in[0,1]$, when $p_{i}$ is input of $t_{j}, \delta_{i j}=1$, when $p_{i}$ is not input of $\mathrm{t}_{\mathrm{j}}, \delta_{\mathrm{ij}}=0, \quad \mathrm{i}=1,2, \ldots, \mathrm{n}, \quad \mathrm{j}=1,2, \ldots, \mathrm{m}$;

$\mathrm{O}=\left(\gamma_{\mathrm{ij}}\right)$ is the rules output matrix from place to transition, $\gamma_{\mathrm{ij}} \in[0,1]$, when $\mathrm{p}_{\mathrm{i}}$ is input of $\mathrm{t}_{\mathrm{j}}, \gamma_{\mathrm{ij}}=1$, when $\mathrm{p}_{\mathrm{i}}$ is not input of $\mathrm{t}_{\mathrm{j}}, \gamma_{\mathrm{ij}}=0, \mathrm{i}=1,2, \ldots, \mathrm{n}, \mathrm{j}=1,2, \ldots, \mathrm{m}$;

$\lambda=\left(\lambda_{1}, \lambda_{2}, \ldots, \lambda_{m}\right), \lambda_{j}$ is the threshold value of transition $\mathrm{t}_{\mathrm{j}}(\mathrm{j}=1,2, \ldots, \mathrm{m})$;

\section{KNOWLEDGE REASONING ALGORITHM OF FUZZY PETRI} NET

According to the thought of MYCIN system, let's define the several following max-algebra operator ${ }^{[4]}$.

Definition 2

$\oplus$ :a $\oplus$ b=c, a,b,c is $\mathrm{N}$-dimensional vector, then $\mathrm{c}_{\mathrm{i}}=\max \left(\mathrm{a}_{\mathrm{i}}, \mathrm{b}_{\mathrm{i}}\right)$;

$\otimes: A \otimes b=d, A$ is the matrix of $n \times m, b$ is m-dimensional vector, $\mathrm{N}$-dimensional vector, then

$$
d_{i}=\max \left(a_{i j \leq \leq m} \cdot \mathrm{b}_{\mathrm{j}}\right)
$$

$\operatorname{neg}(\cdot): \operatorname{neg}(\mathrm{e})=1_{\mathrm{m}}$-e,e is $\mathrm{m}$-dimensional vector, and $1_{\mathrm{m}}$ is m-dimensional vector also, where the all elements are equal to 1 .

Suppose that transition $\mathrm{t}_{\mathrm{j}}$ has $\mathrm{n}$ premise input places and certainty factor of each place are $\mathrm{W}_{\mathrm{j} 1}, \mathrm{~W}_{\mathrm{j} 2}, \ldots, \mathrm{W}_{\mathrm{jn}}$, according to the theory in MYCIN system that"the true value of conjunction expression get the minimum value from the true value of each minor, and the true value of disjunction get the maximum value from it.", so we can calculate the 
certainty factor $\varphi=\min \left(W_{j 1}^{k}, W_{j 2}^{k}, \ldots, W_{j n}^{k}\right)$, where the premise of transition $\mathrm{t}_{\mathrm{j}}$ is true.

Observationally, we can find that the certainty factor $\mathrm{W}$ of place has the following algebra relationship with the premise certainty factor of transition $\varphi$ :

$$
\varphi^{\mathrm{k}}=\operatorname{neg}\left(\mathrm{I}^{\mathrm{T}} \otimes\left(\operatorname{neg}\left(\mathrm{W}^{\mathrm{k}}\right)\right)\right) \text {. }
$$

Setting $\mathrm{k}$ is the inference steps of Fuzzy Petri Net, initial $\mathrm{k}=0$, then the inference algorithm can be described as following steps:

Step 1 Setting $\mathrm{k}=0$;

Step 2 Solving $\mathrm{W}^{\mathrm{k}+1}$ based on

$\mathrm{W}^{\mathrm{k}+1}=\mathrm{W}^{\mathrm{k}} \oplus\left[(\mathrm{O} \cdot \mathrm{CF}) \otimes \varphi^{\mathrm{k}}\right]$;

Step 3 If $\mathrm{W}^{\mathrm{k}+1} \neq \mathrm{W}^{\mathrm{k}}$, then $\mathrm{k}=\mathrm{k}+1$, go to Step 2;

Step 4 If $\mathrm{W}^{\mathrm{k}+1}=\mathrm{W}^{\mathrm{k}}$, then the inference is over.

\section{RESEARCH ON ACCIDENT Evolution Model of COAL AND Gas OUTBURST ON THE BASIS OF FuZZY PETRI NET (AEMCGO-FPN)}

\section{A. Fuzzy-style production rules}

In real-world, many data cannot be expressed by the precise form, so fuzzy-style production rules are adapted to express that fuzzy and uncertain knowledge. Fuzzy-style production rules depict the fuzzy relationship between the multiple propositions.

If $\mathrm{R}$ is a fuzzy production rule system $\mathrm{R}=\{\mathrm{R} 1, \mathrm{R} 2, \ldots$, $\mathrm{Rm}\}$, then $\mathrm{Ri}(\mathrm{i}=1,2, \ldots, \mathrm{m})$ in the form can generally be divided into the following two categories. When $n=1$, the following fuzzy production rules will be simplified as only one premise proposition simple fuzzy production rules .

1) “AND” rule

Ri:IF $d_{1}$ AND $d_{2}$ AND ...AND $d_{n}$ THEN d (CF), $\lambda$

Where $d_{j}(j=1,2, \ldots, n)$ is premise proposition, $d$ is result proposition, $\mathrm{CF}$ is the certainty factor of rules, and $\lambda$ is the threshold value of rule of all prerequisite supported conclusions, which values in $[0,1]$.

2) “OR" rule

$\mathrm{R}_{\mathrm{i}}:$ IF $\mathrm{d}_{1}$ OR $\mathrm{d}_{2}$ OR ... OR $\mathrm{d}_{\mathrm{n}}$ THEN d (CF), $\lambda$

Where $d_{j}(j=1,2, \ldots, n)$ is premise proposition, $d$ is result proposition, $\mathrm{CF}$ is the certainty factor of rules, and $\lambda$ is the threshold value of rule.

B. The establishment of Accident Evolution Model of Coal and Gas Outburst on the Basis of Fuzzy Petri Net (AEMCGO-FPN)

According to the gas outburst mechanism hypothesis and gas geological theories, some researches on coal and gas outburst accident during the production process of coal underground mining show that the coal and gas outburst is mainly by the influence and control of the gas characteristics conferred by the seam and its surrounding geological conditions. This article has proposed an Accident Evolution Model of Coal and Gas Outburst on the Basis of Fuzzy Petri Net (AEMCGO-FPN), which is shown as figure1.

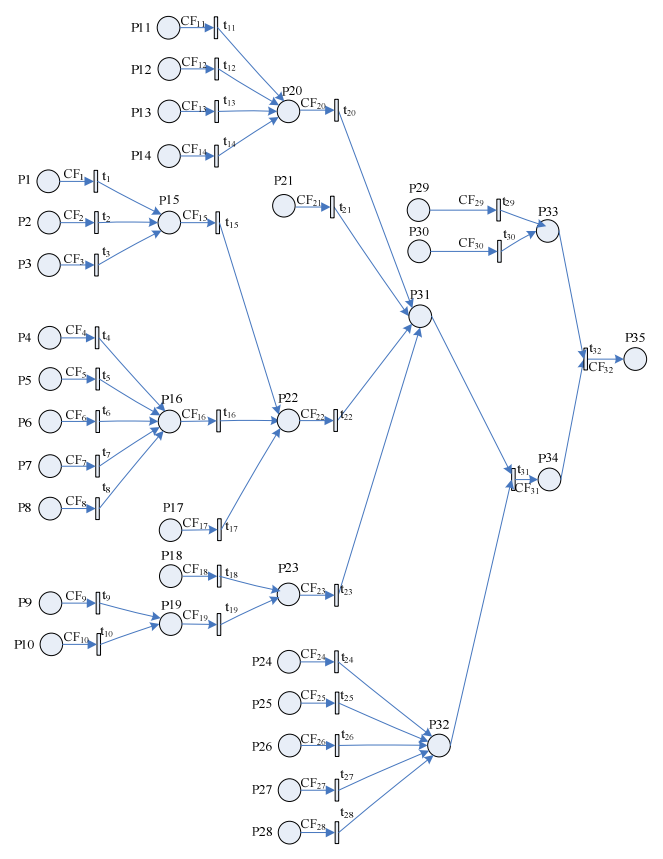

Figure1. Initial AEMCGO-FPN

The reasoning rules of knowledge are as follows:

$\mathrm{R}_{1}$ : IF the disk surrounding rock permeability is poor $\left(\mathrm{p}_{1}\right)$ OR poor hydraulic conductivity of fractured zone $\left(\mathrm{p}_{2}\right)$ OR pressure, pressure -shear closed fault $\left(\mathrm{p}_{3}\right)$ THEN fault structure changes $\left(\mathrm{p}_{15}\right) \quad \mathrm{CF}_{1}, \lambda$;

R2: IF closed fault horst or graben (p4) OR fold complex 、 closed crumpled high coefficient (p5) OR poor ventilation of anticlinal saddle as well as overlying rock (p6) OR synclinal axis office of low permeability coal seam (p7) OR coal seam and tendency to dip suddenly change (p8) THEN fold structure change (p16) $\mathrm{CF}_{4}, \lambda$;

$\mathrm{R}_{3}$ : IF high degree of metamorphism of coal $\left(\mathrm{p}_{9}\right)$ OR seal coal seam gas forming of rock wall and bedrock $\left(\mathrm{p}_{10}\right)$ THEN magmatic intrusion $\left(\mathrm{p}_{19}\right) \quad \mathrm{CF}_{9}, \lambda$;

$\mathrm{R}_{4}$ : IF small coal seam dip $\left(\mathrm{p}_{11}\right)$ OR seam thickness and diversification is large $\left(\mathrm{p}_{12}\right)$ OR the complexity of the coal seam structure $\left(\mathrm{p}_{13}\right)$ OR seam bifurcation, merging $\left(\mathrm{p}_{14}\right)$ THEN coal seams change $\left(\mathrm{p}_{20}\right) \mathrm{CF}_{11, \lambda}$;

$\mathrm{R}_{5}$ : IF fault structure change $\left(\mathrm{p}_{15}\right)$ OR fold structure change $\left(\mathrm{p}_{16}\right)$ or interlayer Slip $\left(\mathrm{p}_{17}\right)$ THEN geological structure change $\left(\mathrm{p}_{22}\right) \quad \mathrm{CF}_{16}, \lambda$;

$\mathrm{R}_{6}$ : IF groundwater activities become more frequent $\left(\mathrm{p}_{18}\right)$ OR lava immersed $\left(\mathrm{p}_{19}\right)$ THEN other geological factors change $\left(\mathrm{p}_{23}\right) \quad \mathrm{CF}_{18}, \lambda$;

$\mathrm{R}_{7}$ : IF coal seams change $\left(\mathrm{p}_{20}\right)$ OR poor permeability of surrounding rock $\left(\mathrm{p}_{21}\right) \mathrm{OR}$ geological structure change $\left(\mathrm{p}_{22}\right)$ OR other geological factors change $\left(\mathrm{p}_{23}\right)$ THEN coal seams, surrounding rock, geological structure and other geological factors change $\left(\mathrm{p}_{31}\right) \quad \mathrm{CF}_{20}, \lambda$;

$\mathrm{R}_{8}$ : IF high index of gas irradiation $\left(\mathrm{p}_{24}\right)$ OR high porosity of coal body $\left(\mathrm{p}_{25}\right)$ OR low intensity of coal $\left(\mathrm{p}_{26}\right)$ OR Strong fragmentation of coal body $\left(\mathrm{p}_{27}\right)$ OR poor ventilation of coal body $\left(\mathrm{p}_{28}\right)$ THEN coal body structure change $\left(\mathrm{p}_{32}\right)$ $\mathrm{CF}_{24}, \lambda$; 
$\mathrm{R}_{9}$ : IF gas content is greater than $10 \mathrm{~m}^{3} / \mathrm{t}\left(\mathrm{p}_{29}\right)$ OR gas pressure is greater than $0.75 \mathrm{MPa}\left(\mathrm{p}_{30}\right)$ THEN gas changes $\left(\mathrm{p}_{33}\right) \mathrm{CF}_{29}, \lambda$;

$\mathrm{R}_{10}$ : IF coal seams, surrounding rock, geological structure and other geological factors change $\left(\mathrm{p}_{31}\right)$ AND coal body structure change $\left(\mathrm{p}_{32}\right)$ THEN geological changes $\left(\mathrm{p}_{34}\right) \mathrm{CF}_{31}, \lambda$;

$\mathrm{R}_{11}$ : IF gas changes $\left(\mathrm{p}_{33}\right)$ AND geological changes $\left(\mathrm{p}_{34}\right)$ THEN coal and gas outburst accident occurred $\left(\mathrm{p}_{35}\right)$ CF32, $\lambda$;

\section{REASONING AND ANALYSIS OF AEMCGO-FPN AFTER REDUCTION}

Figure 1 above shows that the destination place in this instance is $\left\{p_{35}\right\}$, and initial places set are $\left\{p_{1}, p_{2}, p_{3}, p_{4}\right.$. $\mathrm{p}_{5}, \mathrm{p}_{6}, \mathrm{p}_{8}, \mathrm{p}_{9}, \mathrm{p}_{10}, \mathrm{p}_{11}, \mathrm{p}_{12}, \mathrm{p}_{13}, \mathrm{p}_{14}, \mathrm{p}_{17}, \mathrm{p}_{18}, \mathrm{p}_{21}, \mathrm{p}_{24}, \mathrm{p}_{25}, \mathrm{p}_{26}$, $\left.\mathrm{p}_{27}, \mathrm{p}_{28}, \mathrm{p}_{29}, \mathrm{p}_{30}\right\}$. According to the Gas geological conditions in Luling Coal Mine of Huaibei Mining Group Company and actual material of coal and gas outburst occurred and recorded in 8 and 9 coal seam since the establishment of mine [5]. Setting that the initial certainty factor of initial places of this AEMCGO-FPN is $\mathrm{W}=(0.04$ $\begin{array}{llllllllll}0.01 & 0.23 & 0.01 & 0.01 & 0.08 & 0.08 & 0.01 & 0.01 & 0.01 & 0.01\end{array}$ $\begin{array}{lllllllllllll}0.3 & 0.08 & 0.38 & 0 & 0 & 0.15 & 0.01 & 0 & 0 & 0.15 & 0 & 0 & 0.01\end{array}$ $\left.\begin{array}{lllllllllll}0.01 & 0.42 & 0.42 & 0.42 & 0.15 & 0.19 & 0 & 0 & 0 & 0 & 0\end{array}\right)$.In terms of the critical coefficient of minimal cut sets in coal and gas outburst accident of Luling Coal Mine inferred from article [5], setting the transition certainty factor of this model is equal to CF: $\left\{\begin{array}{lllllll}0.7 & 0.6 & 0.9 & 0.6 & 0.6 & 0.7 & 0.6\end{array}\right.$ $\begin{array}{lllllllllll}0.6 & 0.6 & 0.6 & 0.6 & 0.9 & 0.85 & 0.95 & 0.7 & 0.8 & 0.9 & 0.6\end{array}$ $\begin{array}{lllllllllll}0.6 & 0.95 & 0.9 & 0.9 & 0.6 & 0.7 & 0.7 & 0.95 & 0.95 & 0.95 & 0.95\end{array}$ $\begin{array}{lll}1.0 & 0.9 & 0.9\end{array}$.

And then the Reach ability Set (RS), Immediate Reach ability Set (IRS) and Adjacent Place set (APS) for each place can be established, shown as Table 1, Table2.

In this instance, setting the threshold values $\lambda$ of all transition $t_{i}(i=1,2, \ldots, 32)$ is equal to 0.15 . And then the net can be reduced on the basis of Fuzzy Petri net reduction algorithm by use of $\operatorname{RS}\left(\mathrm{P}_{\mathrm{i}}\right), \operatorname{IRS}\left(\mathrm{P}_{\mathrm{i}}\right)$ and $\mathrm{AP}$ $\left(P_{i}\right)$. The AEMCGO-FPN after reduction is shown as figure.2.

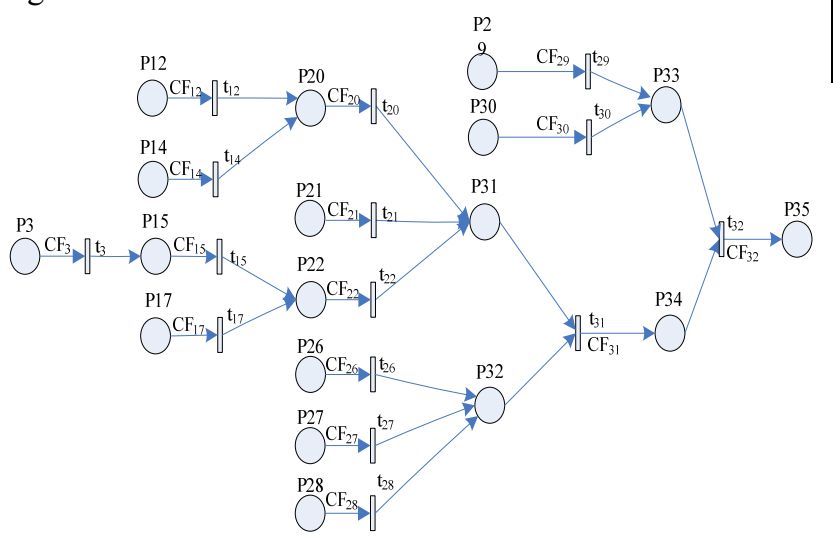

Figure2. AEMCGO-FPN after reduction
Table 1:IRS $\left(\mathrm{P}_{\mathrm{i}}\right)$ and $\operatorname{RS}\left(\mathrm{P}_{\mathrm{i}}\right)$

Table 2: $\operatorname{AP}\left(\mathrm{P}_{\mathrm{ij}}\right)$

\begin{tabular}{|c|c|c|}
\hline $\begin{array}{c}\mathrm{Pla} \\
\text { ce } \\
\mathrm{P}_{\mathrm{i}}\end{array}$ & $\begin{array}{l}\text { IRS } \\
\left(P_{i}\right)\end{array}$ & $\mathrm{RS}\left(\mathrm{P}_{\mathrm{i}}\right)$ \\
\hline $\mathrm{P}_{1}$ & $\left\{\mathrm{P}_{15}\right\}$ & $\left\{\mathrm{P}_{15}, \mathrm{P}_{22}, \mathrm{P}_{31}, \mathrm{P}_{34}, \mathrm{P}_{35}\right\}$ \\
\hline $\mathrm{P}_{2}$ & $\left\{\mathrm{P}_{15}\right\}$ & $\left\{\mathrm{P}_{15}, \mathrm{P}_{22}, \mathrm{P}_{31}, \mathrm{P}_{34}, \mathrm{P}_{35}\right\}$ \\
\hline $\mathrm{P}_{3}$ & $\left\{\mathrm{P}_{15}\right\}$ & $\left\{\mathrm{P}_{15}, \mathrm{P}_{22}, \mathrm{P}_{31}, \mathrm{P}_{34}, \mathrm{P}_{35}\right\}$ \\
\hline $\mathrm{P}_{4}$ & $\left\{\mathrm{P}_{16}\right\}$ & $\left\{\mathrm{P}_{16}, \mathrm{P}_{22}, \mathrm{P}_{31}, \mathrm{P}_{34}, \mathrm{P}_{35}\right\}$ \\
\hline $\mathrm{P}_{5}$ & $\left\{\mathrm{P}_{16}\right\}$ & $\left\{\mathrm{P}_{16}, \mathrm{P}_{22}, \mathrm{P}_{31}, \mathrm{P}_{34}, \mathrm{P}_{35}\right\}$ \\
\hline $\mathrm{P}_{6}$ & $\left\{\mathrm{P}_{16}\right\}$ & $\left\{\mathrm{P}_{16}, \mathrm{P}_{22}, \mathrm{P}_{31}, \mathrm{P}_{34}, \mathrm{P}_{35}\right\}$ \\
\hline $\mathrm{P}_{7}$ & $\left\{\mathrm{P}_{16}\right\}$ & $\left\{\mathrm{P}_{16}, \mathrm{P}_{22}, \mathrm{P}_{31}, \mathrm{P}_{34}, \mathrm{P}_{35}\right\}$ \\
\hline $\mathrm{P}_{8}$ & $\left\{\mathrm{P}_{16}\right\}$ & $\left\{\mathrm{P}_{16}, \mathrm{P}_{22}, \mathrm{P}_{31}, \mathrm{P}_{34}, \mathrm{P}_{35}\right\}$ \\
\hline $\mathrm{P}_{9}$ & $\left\{\mathrm{P}_{19}\right\}$ & $\left\{\mathrm{P}_{19}, \mathrm{P}_{23}, \mathrm{P}_{31}, \mathrm{P}_{34}, \mathrm{P}_{35}\right\}$ \\
\hline $\mathrm{P}_{10}$ & $\left\{\mathrm{P}_{19}\right\}$ & $\left\{\mathrm{P}_{19}, \mathrm{P}_{23}, \mathrm{P}_{31}, \mathrm{P}_{34}, \mathrm{P}_{35}\right\}$ \\
\hline $\mathrm{P}_{11}$ & $\left\{\mathrm{P}_{20}\right\}$ & $\left\{\mathrm{P}_{20}, \mathrm{P}_{31}, \mathrm{P}_{34}, \mathrm{P}_{35}\right\}$ \\
\hline $\mathrm{P}_{12}$ & $\left\{\mathrm{P}_{20}\right\}$ & $\left\{\mathrm{P}_{20}, \mathrm{P}_{31}, \mathrm{P}_{34}, \mathrm{P}_{35}\right\}$ \\
\hline $\mathrm{P}_{13}$ & $\left\{\mathrm{P}_{20}\right\}$ & $\left\{\mathrm{P}_{20}, \mathrm{P}_{31}, \mathrm{P}_{34}, \mathrm{P}_{35}\right\}$ \\
\hline $\mathrm{P}_{14}$ & $\left\{\mathrm{P}_{20}\right\}$ & $\left\{\mathrm{P}_{20}, \mathrm{P}_{31}, \mathrm{P}_{34}, \mathrm{P}_{35}\right\}$ \\
\hline $\mathrm{P}_{15}$ & $\left\{\mathrm{P}_{22}\right\}$ & $\left\{\mathrm{P}_{22}, \mathrm{P}_{31}, \mathrm{P}_{34}, \mathrm{P}_{35}\right\}$ \\
\hline $\mathrm{P}_{16}$ & $\left\{\mathrm{P}_{22}\right\}$ & $\left\{\mathrm{P}_{22}, \mathrm{P}_{31}, \mathrm{P}_{34}, \mathrm{P}_{35}\right\}$ \\
\hline $\mathrm{P}_{17}$ & $\left\{\mathrm{P}_{22}\right\}$ & $\left\{\mathrm{P}_{22}, \mathrm{P}_{31}, \mathrm{P}_{34}, \mathrm{P}_{35}\right\}$ \\
\hline $\mathrm{P}_{18}$ & $\left\{\mathrm{P}_{23}\right\}$ & $\left\{\mathrm{P}_{23}, \mathrm{P}_{31}, \mathrm{P}_{34}, \mathrm{P}_{35}\right\}$ \\
\hline $\mathbf{P}_{19}$ & $\left\{\mathrm{P}_{23}\right\}$ & $\left\{\mathrm{P}_{23}, \mathrm{P}_{31}, \mathrm{P}_{34}, \mathrm{P}_{35}\right\}$ \\
\hline $\mathrm{P}_{20}$ & $\left\{\mathrm{P}_{31}\right\}$ & $\left\{\mathrm{P}_{31}, \mathrm{P}_{34}, \mathrm{P}_{35}\right\}$ \\
\hline $\mathrm{P}_{21}$ & $\left\{\mathrm{P}_{31}\right\}$ & $\left\{\mathrm{P}_{31}, \mathrm{P}_{34}, \mathrm{P}_{35}\right\}$ \\
\hline $\mathrm{P}_{22}$ & $\left\{\mathrm{P}_{31}\right\}$ & $\left\{\mathrm{P}_{31}, \mathrm{P}_{34}, \mathrm{P}_{35}\right\}$ \\
\hline $\mathrm{P}_{23}$ & $\left\{\mathrm{P}_{31}\right\}$ & $\left\{\mathrm{P}_{31}, \mathrm{P}_{34}, \mathrm{P}_{35}\right\}$ \\
\hline $\mathrm{P}_{24}$ & $\left\{\mathrm{P}_{32}\right\}$ & $\left\{\mathrm{P}_{32}, \mathrm{P}_{34}, \mathrm{P}_{35}\right\}$ \\
\hline $\mathrm{P}_{25}$ & $\left\{\mathrm{P}_{32}\right\}$ & $\left\{\mathrm{P}_{32}, \mathrm{P}_{34}, \mathrm{P}_{35}\right\}$ \\
\hline $\mathrm{P}_{26}$ & $\left\{\mathrm{P}_{32}\right\}$ & $\left\{\mathrm{P}_{32}, \mathrm{P}_{34}, \mathrm{P}_{35}\right\}$ \\
\hline $\mathrm{P}_{27}$ & $\left\{\mathrm{P}_{32}\right\}$ & $\left\{\mathrm{P}_{32}, \mathrm{P}_{34}, \mathrm{P}_{35}\right\}$ \\
\hline $\mathrm{P}_{28}$ & $\left\{\mathrm{P}_{32}\right\}$ & $\left\{\mathrm{P}_{32}, \mathrm{P}_{34}, \mathrm{P}_{35}\right\}$ \\
\hline $\mathrm{P}_{29}$ & $\left\{\mathrm{P}_{33}\right\}$ & $\left\{\mathrm{P}_{33}, \mathrm{P}_{35}\right\}$ \\
\hline $\mathrm{P}_{30}$ & $\left\{\mathrm{P}_{33}\right\}$ & $\left\{\mathrm{P}_{33}, \mathrm{P}_{35}\right\}$ \\
\hline $\mathrm{P}_{31}$ & $\left\{\mathrm{P}_{34}\right\}$ & $\left\{\mathrm{P}_{34}, \mathrm{P}_{35}\right\}$ \\
\hline $\mathrm{P}_{32}$ & $\left\{\mathrm{P}_{34}\right\}$ & $\left\{\mathrm{P}_{34}, \mathrm{P}_{35}\right\}$ \\
\hline $\mathrm{P}_{33}$ & $\left\{\mathrm{P}_{35}\right\}$ & $\left\{\mathrm{P}_{35}\right\}$ \\
\hline $\mathrm{P}_{34}$ & $\left\{\mathrm{P}_{35}\right\}$ & $\left\{\mathrm{P}_{35}\right\}$ \\
\hline $\mathrm{P}_{35}$ & $\Phi$ & $\Phi$ \\
\hline
\end{tabular}

\begin{tabular}{|c|c|c|}
\hline $\begin{array}{c}\text { Place } \\
\mathrm{P}_{\mathrm{i}}\end{array}$ & $\begin{array}{c}\mathrm{Place}_{\mathrm{i}} \\
\mathrm{P}_{\mathrm{i}}\end{array}$ & $\mathrm{AP}\left(\mathrm{P}_{\mathrm{ij}}\right)$ \\
\hline $\mathrm{P}_{1}$ & $\left\{\mathrm{P}_{15}\right\}$ & $\Phi$ \\
\hline $\mathrm{P}_{2}$ & $\left\{\mathrm{P}_{15}\right\}$ & $\Phi$ \\
\hline $\mathrm{P}_{3}$ & $\left\{\mathrm{P}_{15}\right\}$ & $\Phi$ \\
\hline $\mathrm{P}_{4}$ & $\left\{\mathrm{P}_{16}\right\}$ & $\Phi$ \\
\hline $\mathrm{P}_{5}$ & $\left\{\mathrm{P}_{16}\right\}$ & $\Phi$ \\
\hline $\mathrm{P}_{6}$ & $\left\{\mathrm{P}_{16}\right\}$ & $\Phi$ \\
\hline $\mathrm{P}_{7}$ & $\left\{\mathrm{P}_{16}\right\}$ & $\Phi$ \\
\hline $\mathrm{P}_{8}$ & $\left\{\mathrm{P}_{16}\right\}$ & $\Phi$ \\
\hline $\mathrm{P}_{9}$ & $\left\{\mathrm{P}_{19}\right\}$ & $\Phi$ \\
\hline $\mathrm{P}_{10}$ & $\left\{\mathrm{P}_{19}\right\}$ & $\Phi$ \\
\hline $\mathrm{P}_{11}$ & $\left\{\mathrm{P}_{20}\right\}$ & $\Phi$ \\
\hline $\mathrm{P}_{12}$ & $\left\{\mathrm{P}_{20}\right\}$ & $\Phi$ \\
\hline $\mathrm{P}_{13}$ & $\left\{\mathrm{P}_{20}\right\}$ & $\Phi$ \\
\hline $\mathrm{P}_{14}$ & $\left\{\mathrm{P}_{20}\right\}$ & $\Phi$ \\
\hline $\mathrm{P}_{15}$ & $\left\{\mathrm{P}_{22}\right\}$ & $\Phi$ \\
\hline $\mathrm{P}_{16}$ & $\left\{\mathrm{P}_{22}\right\}$ & $\Phi$ \\
\hline $\mathrm{P}_{17}$ & $\left\{\mathrm{P}_{22}\right\}$ & $\Phi$ \\
\hline $\mathrm{P}_{18}$ & $\left\{\mathrm{P}_{23}\right\}$ & $\Phi$ \\
\hline $\mathrm{P}_{19}$ & $\left\{\mathrm{P}_{23}\right\}$ & $\Phi$ \\
\hline $\mathrm{P}_{20}$ & $\left\{\mathrm{P}_{31}\right\}$ & $\Phi$ \\
\hline $\mathrm{P}_{21}$ & $\left\{\mathrm{P}_{31}\right\}$ & $\Phi$ \\
\hline $\mathrm{P}_{22}$ & $\left\{\mathrm{P}_{31}\right\}$ & $\Phi$ \\
\hline $\mathrm{P}_{23}$ & $\left\{\mathrm{P}_{31}\right\}$ & $\Phi$ \\
\hline $\mathrm{P}_{24}$ & $\left\{\mathrm{P}_{32}\right\}$ & $\Phi$ \\
\hline $\mathrm{P}_{25}$ & $\left\{\mathrm{P}_{32}\right\}$ & $\Phi$ \\
\hline $\mathrm{P}_{26}$ & $\left\{\mathrm{P}_{32}\right\}$ & $\Phi$ \\
\hline $\mathrm{P}_{27}$ & $\left\{\mathrm{P}_{32}\right\}$ & $\Phi$ \\
\hline $\mathrm{P}_{28}$ & $\left\{\mathrm{P}_{32}\right\}$ & $\Phi$ \\
\hline $\mathrm{P}_{29}$ & $\left\{\mathrm{P}_{33}\right\}$ & $\Phi$ \\
\hline $\mathrm{P}_{30}$ & $\left\{\mathrm{P}_{35}\right\}$ & $\Phi$ \\
\hline $\mathrm{P}_{31}$ & $\left\{\mathrm{P}_{34}\right\}$ & $\left\{\mathrm{P}_{32}\right\}$ \\
\hline $\mathrm{P}_{32}$ & $\left\{\mathrm{P}_{34}\right\}$ & $\left\{\mathrm{P}_{31}\right\}$ \\
\hline $\mathrm{P}_{33}$ & $\left\{\mathrm{P}_{35}\right\}$ & $\{\mathrm{P} 34$ \\
\hline $\mathrm{P}_{34}$ & $\left\{\mathrm{P}_{35}\right\}$ & $\left\{\mathrm{P}_{33}\right\}$ \\
\hline $\mathrm{P}_{35}$ & $\Phi_{1}$ & $\Phi$ \\
\hline & & \\
\hline
\end{tabular}

Thereafter, the AEMCGO-FPN after reduction can be positively single-stage fuzzy inferred with great algebra ${ }^{[6]}$. We can get CF' directly from the AEMCGO-FPN after reduction.

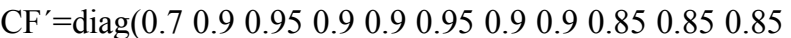
$0.91 .00 .90 .9)$ 


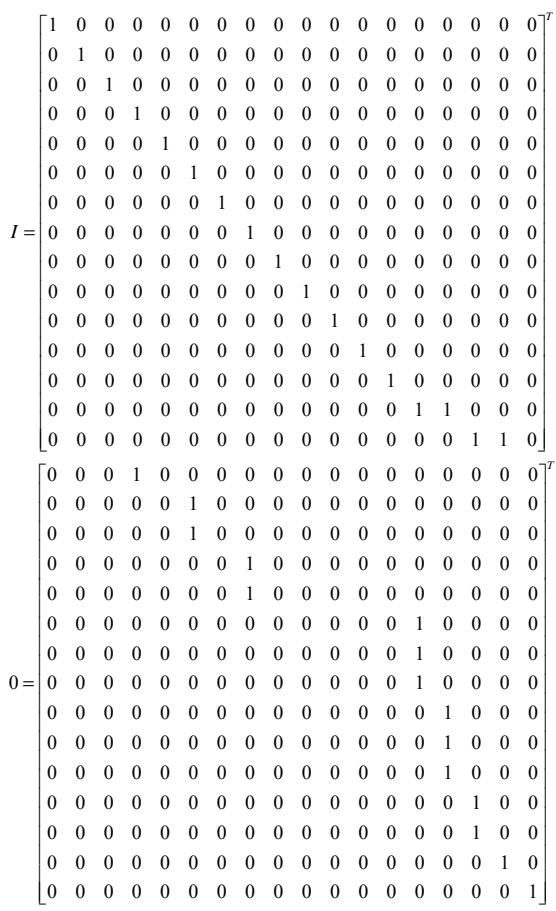

The initial certainty factor of starting places after reduction is $\mathrm{W}^{\prime}=\left(\begin{array}{llllllllll}0.23 & 0.3 & 0.38 & 0 & 0.15 & 0 & 0.15 & 0 & 0.42 & 0.42\end{array}\right.$ $\left.\begin{array}{llllllll}0.42 & 0.15 & 0.19 & 0 & 0 & 0 & 0 & 0\end{array}\right)^{\mathrm{T}}$

We can obtain by the single-stage forward fuzzy reasoning algorithm:

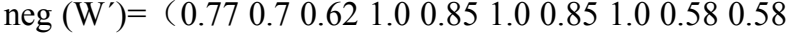
$0.580 .850 .811 .01 .01 .01 .01 .0)^{\mathrm{T}}$

$$
\varphi^{0}=\operatorname{neg}\left(\mathrm{I}^{\mathrm{T}} \otimes\left(\operatorname{neg}\left(\mathrm{W}^{\prime}\right)\right)\right)=\left(\begin{array}{llllll}
0.23 & 0.3 & 0.38 & 0 & 0.15 & 0
\end{array}\right.
$$

$0.1500 .420 .420 .420 .150 .19000)^{\mathrm{T}}$

First round: $\mathrm{W}^{\prime 1}=\mathrm{W}^{\prime} \oplus\left[(\mathrm{O} \cdot \mathrm{CF}) \otimes \varphi^{0}\right]=\left(\begin{array}{lll}0.23 & 0.3 & 0.38\end{array}\right.$ $\begin{array}{llllllllll}0.161 & 0.15 & 0.361 & 0.15 & 0.135 & 0.42 & 0.42 & 0.42 & 0.15 & 0.19\end{array}$ $\left.\begin{array}{lllll}0.072 & 0.357 & 0.19 & 0 & 0\end{array}\right)^{\mathrm{T}}$

Second round: $\mathrm{W}^{\prime 2}=\mathrm{W}^{\prime 1} \oplus\left[(\mathrm{O} \cdot \mathrm{CF}) \otimes \varphi^{1}\right]=\left(\begin{array}{ll}0.23 & 0.3\end{array}\right.$ $\begin{array}{llllllllllll}0.38 & 0.161 & 0.15 & 0.361 & 0.15 & 0.145 & 0.42 & 0.42 & 0.42 & 0.15 & 0.19\end{array}$ $\left.\begin{array}{lllll}0.343 & 0.357 & 0.19 & 0.065 & 0\end{array}\right)^{\mathrm{T}}$

Third round: $\mathrm{W}^{\prime 3}=\mathrm{W}^{\prime 2} \oplus\left[(\mathrm{O} \cdot \mathrm{CF}) \otimes \varphi^{2}\right]=\left(\begin{array}{cc}0.23 & 0.3\end{array}\right.$ $\begin{array}{lllllllllll}0.38 & 0.161 & 0.15 & 0.361 & 0.15 & 0.145 & 0.42 & 0.42 & 0.42 & 0.15 & 0.19\end{array}$ $\left.\begin{array}{llllll}0.343 & 0.357 & 0.19 & 0.309 & 0.059\end{array}\right)^{\mathrm{T}}$

Fourth round: $\mathrm{W}^{\prime 4}=\mathrm{W}^{\prime 3} \oplus\left[(\mathrm{O} \cdot \mathrm{CF}) \otimes \varphi^{3}\right]=\left(\begin{array}{ll}0.23 & 0.3\end{array}\right.$ $\begin{array}{llllllllllll}0.38 & 0.161 & 0.15 & 0.361 & 0.15 & 0.145 & 0.42 & 0.42 & 0.42 & 0.15 & 0.19\end{array}$ $\begin{array}{llllll}0.343 & 0.357 & 0.19 & 0.309 & 0.171)^{\mathrm{T}}\end{array}$

Fifth round: $\mathrm{W}^{\prime 5}=\mathrm{W}^{\prime 4} \oplus\left[(\mathrm{O} \cdot \mathrm{CF}) \otimes \varphi^{4}\right]=\left(\begin{array}{lll}0.23 & 0.3\end{array}\right.$ $\begin{array}{lllllllllll}0.38 & 0.161 & 0.15 & 0.361 & 0.15 & 0.145 & 0.42 & 0.42 & 0.42 & 0.15 & 0.19\end{array}$ $0.3430 .3570 .190 .3090 .171)^{\mathrm{T}}$

Because of $\mathrm{W}^{4}=\mathrm{W}^{5}$, the reasoning process is over, and the finial state of places in AEMCGO-FPN is ( 0.230 .30 .38 $\begin{array}{llllllllll}0.161 & 0.15 & 0.361 & 0.15 & 0.145 & 0.42 & 0.42 & 0.42 & 0.15 & 0.19\end{array}$ $\left.\begin{array}{lllll}0.343 & 0.357 & 0.19 & 0.309 & 0.171\end{array}\right)$

\section{CONCLUSION}

It can be seen from the reasoning conclusion that the certainty factor of the "coal and gas outburst" in this circumstance is 0.171 , and the certainty factor of the accident caused by gas factor is 0.19 , and by geological factor changing is 0.309 . From the point of the reasoning processes, the risk the "coal and gas outburst accident" evolves from the small risk factors, including pressure, pressure-shear closed fault, large seam thickness and diversification, seam bifurcation, merge, poor ventilation of wall rock, low intensity of coal body, strong coal crushing, poor ventilation of coal body etc. into significant changes in coal structure and coal seam, wall rock, geological structure and other geological factors. Therefore, under the cooperation of some above risk factors, thus it evolves into geological significant changes. In this case, coupled with the gas changing such as the gas content is greater than $10 \mathrm{~m}^{3} / \mathrm{t}$, gas pressure is greater than $0.75 \mathrm{MPa}$, it will evolve into coal and gas outburst.

Reasoning has been carried out for five rounds. Each round of reasoning represents a step of accident evolution of coal and gas outburst. During the evolution process of this case, changes of certainty factor in each place reflect the status and role of the various in each aspect of coal and gas outburst accident evolution. For example, in the initial conditions, the certainty factor of these geological factors is equal to 0 , such as coal body structure, coal seam, wall rock, geological construction and other geological factors change. At this time, they will not cause coal and gas outburst. However, their certainty factor has changed into 0.357 and 0.343 in the accident process of evolution, which illustrate that the degree to cause risk has increased. It can be seen from the result data that the factors such as low intensity of coal, strong coal crushing and poor ventilation of coal body has close relationship with coal and gas outburst accident.

\section{ACKNOWLEDGMENT}

The authors would like to thank Shugang Li and Haifei Lin for their helpful comments and suggestions on earlier version of the manuscript. The authors also wish to thank anonymous referees for their feedback and comments.

\section{REFERENCES}

[1] Xin Yan, Hua Fu. Prediction of coal and gas outburst based on data fusion and case-based reasoning, Journal of Southeast University (Natural Science Edition), 41(z1):59-63,2011.

[2] Ruilin Zhang,Research on Prognosticating Coal and Gas Outburst in Area Coverage Applying Present-day Information Techniques[D]. Chungking University, pp.45,2004.

[3]Xin-gui He,Fuzzy Petri net[J], Journal of Computers,vol12,pp.946$950,1994$.

[4] Tazaki E,Yoshida K.A fuzzy Petri net model for approximate rea-soning and its application to medical diagnosis[J],IEEE International Conference on System,Man and Cybernetics,vol 1,pp.627-631,( 1992).

[5]Rui-lin Zhang. Reseach into the factors of controlling gas-outburst by FTA [J]. China Mining Magazine,vol 5,2005.

[6]Min Guo, A safety assessment research on factors that may lead to coal/gas outbursts [J], China Coal,vol 3,2010. 